\title{
EXAMPLES OF RANK 3 PRODUCT ACTION TRANSITIVE DECOMPOSITIONS
}

\author{
GEOFFREY PEARCE
}

\begin{abstract}
A transitive decomposition is a pair $(\Gamma, \mathcal{P})$ where $\Gamma$ is a graph and $\mathcal{P}$ is a partition of the arc set of $\Gamma$ such that there is a subgroup of automorphisms of $\Gamma$ which leaves $\mathcal{P}$ invariant and transitively permutes the parts in $\mathcal{P}$. In an earlier paper we gave a characterisation of $G$-transitive decompositions where $\Gamma$ is the graph product $K_{m} \times K_{m}$ and $G$ is a rank 3 group of product action type. This characterisation showed that every such decomposition arose from a 2-transitive decomposition of $K_{m}$ via one of two general constructions. Here we use results of Sibley to give an explicit classification of those which arise from 2-transitive edge-decompositions of $K_{m}$.
\end{abstract}

\section{INTRODUCTION}

A $G$-transitive decomposition is a pair $(\Gamma, \mathcal{P})$ where $\Gamma$ is a graph, $\mathcal{P}$ is a partition of its arc set $A \Gamma$, and $G$ is a subgroup of Aut $\Gamma$ such that

(i) for all $P \in \mathcal{P}$ and $g \in G$ we have $P^{g} \in \mathcal{P}$; and

(ii) for all $P, P^{\prime} \in \mathcal{P}$, there exists $g \in G$ with $P^{g}=P^{\prime}$.

Usually we require that $|\mathcal{P}|>1$; however we may sometimes allow $|\mathcal{P}|=1$, in which case we call the decomposition degenerate. We say that $\mathcal{P}$ is symmetric if for any $P \in \mathcal{P}$ and $(\alpha, \beta) \in P$ we have $(\beta, \alpha) \in P$ also. In this case we may view $\mathcal{P}$ as an edge-decomposition of $\Gamma$ by identifying the pair $(\alpha, \beta),(\beta, \alpha)$ of arcs with the edge $\{\alpha, \beta\}$.

Transitive decompositions generalise a number of other mathematical structures, including homogeneous factorisations [10, 11], line transitive partial linear spaces [6], and 2-transitive 1-factorisations of complete graphs [4]; and they are related to 2-transitive symmetric graph designs [3] and 2-transitive symmetric association schemes [2]. Explanations of several of these relationships can be found in [13], [14] and [15]. The last of these papers ([15]) is a characterisation by Sibley of all $G$-transitive decompositions where $G$ is a 2-transitive (rank 2) permutation group. In [1] we extended Sibley's work to the rank 3 case; in particular, we gave a characterisation of $G$-transitive decompositions where $G$ is a primitive rank 3 group of product action type. In doing so we generalised a classification of rank 3 product action partial linear spaces by Devillers [6].

This paper concerns the $G$-transitive decompositions studied in [1]. We may assume that such a rank 3 group $G$ of product action type is contained in $H$ ? $S_{2}$ where $H$ is a 2-transitive group of almost simple type (see for example [1, Lemma 3.4]). The characterisation in [1] amounted to showing that any such transitive decomposition can be obtained using one of several explicit 'product' constructions. These constructions involved an $H$-transitive decomposition $\left(K_{m}, \mathcal{Q}\right)$, and all such $\left(K_{m}, \mathcal{Q}\right)$ with a symmetric partition $\mathcal{Q}$ are classified in [15]. However, [1, Construction 2.10] (which we re-state in Construction 1.3) also involved an $H$-invariant refinement $\mathcal{R}$ of the partition $\mathcal{Q}$, and a

2000 Mathematics Subject Classification. Primary 20B15, 05C25, 05 E20.

Key words and phrases. transitive decomposition, rank 3 primitive group, product action type.

The author gratefully acknowledges advice and assistance from his Ph.D. supervisors, Cheryl E. Praeger and John Bamberg, in the writing of this paper. The author was supported by an Australian Postgraduate Award. 
'twisting' function $\varphi$. The purpose of this paper is to find all possible $\mathcal{R}$ and $\varphi$ when $\mathcal{Q}$ is symmetric, and thereby give a more explicit description of this class of rank 3 product action transitive edge-decompositions.

Throughout the paper we use the following notation.

\section{Notation 1.1.}

(a) $\Gamma$ is the graph product $\Delta \times \Delta$, where $\Delta=K_{m}$ with vertex set $\Omega_{0}$ and $\left|\Omega_{0}\right|=m$. Here $V \Gamma=\Omega_{0} \times \Omega_{0}$ and $((\alpha, \gamma),(\beta, \delta)) \in A \Gamma$ whenever $(\alpha, \beta)$ and $(\gamma, \delta)$ are both arcs of $K_{m}$ (that is, whenever both $\alpha \neq \beta$ and $\gamma \neq \delta$ ).

(b) $G \leq H<S_{2} \leq$ Aut $\Gamma$ in product action on $\Omega_{0} \times \Omega_{0}$ where $H$ is almost simple and 2-transitive on $\Omega_{0}$. We let $T=\operatorname{P\Gamma L}(2,8)$ if $\left(H,\left|\Omega_{0}\right|\right)=(\operatorname{P\Gamma L}(2,8), 28)$, and otherwise we let $T=\operatorname{Soc}(H)$, the unique minimal normal subgroup of $H$. Note that $T$ is 2-transitive on $\Omega_{0}$.

(c) $(\Gamma, \mathcal{P})$ is a $G$-transitive decomposition and $\mathcal{P}=\mathcal{P}(\mathcal{T}, \mathcal{R}, \varphi)$ where $\mathcal{T}=(\Delta, \mathcal{Q})$ is an $H$-transitive decomposition, $\mathcal{R}$ is a proper $H$-invariant refinement of $\mathcal{Q}$, and $\varphi$ is a 'twisting' homomorphism as in Construction 1.3.

Our main result is the following.

Theorem 1.2. Let $G, \Gamma, m, \mathcal{P}(\mathcal{T}, \mathcal{R}, \varphi)$ and $\mathcal{Q}$ be as in Notation 1.1 , and let $(\alpha, \beta)$ be an arc in $Q_{0} \in \mathcal{Q}$. Then

(i) there exist subgroups $L$ and $M$ with $T_{(\alpha, \beta)} \leq M \triangleleft L \leq T$ and $T_{\{\alpha, \beta\}} \leq L$, and $\varphi_{0} \in \operatorname{Aut}(L / M)$ such that $L, M, \varphi_{0}$ determine $\mathcal{T}, \mathcal{R}, \varphi$; and

(ii) $L, M$ are as in Table 1 or 2 .

Remark. Lemma 3.1 describes explicitly how $L, M$ and $\varphi_{0}$ determine $\mathcal{T}, \mathcal{R}$ and $\varphi$.

\begin{tabular}{|l|l|c|l|l|}
\hline & $T$ & $m$ & $L$ & $M$ \\
\hline (i) & Any 2-t group & - & $T_{\{\alpha, \beta\}}$ & $T_{(\alpha, \beta)}$ \\
(ii) & $A_{7}$ & 15 & Line stabiliser (induces $\left.S_{3}\right)$ & Induces $A_{3}, T_{(\alpha, \beta)}$ \\
(iii) & $\operatorname{PSL}(2,7)$ & 8 & 1-factor stabiliser $\cong S_{4}$ & $A_{4}$ \\
(iv) & $\operatorname{PSL}(2,5)$ & 6 & 1-factor stabiliser $\cong A_{4}$ & $T_{\{\alpha, \beta\}} \cong V_{4}$ \\
(v) & $\operatorname{PSU}(3,3)$ & 28 & $T_{Q}$ from Table 3, Case 8 & $T_{\{\alpha, \beta\}}$ \\
(vi) & $\operatorname{PSL}(a, 2), a \geq 3$ & $2^{a}-1$ & Line stabiliser (induces $\left.S_{3}\right)$ & Induces $A_{3}, T_{(\alpha, \beta)}$ \\
(vii) & $\operatorname{PSL}(a, 3), a \geq 3$ & $\frac{3^{a}-1}{2}$ & $T_{Q}$ from Table 3, Case 6 & $T_{\{\alpha, \beta\}}$ \\
\hline
\end{tabular}

TABLE 1. $T \neq \operatorname{P\Gamma L}(2,8)$

Below is a version of [1, Construction 2.10]. Given subsets $R$ and $R^{\prime}$ of $A \Delta$ we write $R \times$ graph $R^{\prime}$ to denote the subset

$$
\left\{((\alpha, \gamma),(\beta, \delta)) \mid(\alpha, \beta) \in R,(\gamma, \delta) \in R^{\prime}\right\}
$$

of $A(\Delta \times \Delta)$. A transitive permutation group is called regular if each point stabiliser is trivial.

Construction 1.3. Let $\mathcal{T}=(\Delta, \mathcal{Q})$ be a (possibly degenerate) $H$-transitive decomposition, let $\mathcal{R}$ be a proper $H$-invariant refinement of $\mathcal{Q}$, and let $\Gamma=\Delta \times \Delta$.

Let the parts in $\mathcal{Q}$ be denoted by $Q_{0}, Q_{1}, \ldots, Q_{s-1}$, and for each $Q_{i} \in \mathcal{Q}$ let $\mathcal{R}_{Q_{i}}$ denote the set $\left\{R \in \mathcal{R} \mid R \subset Q_{i}\right\}$. Assume that the permutation group $H_{Q_{0}}^{\mathcal{R}_{Q_{0}}}$ induced by $H_{Q_{0}}$ on $\mathcal{R}_{Q_{0}}$ is regular, and let $\varphi$ be an element of $\operatorname{Sym}\left(\mathcal{R}_{Q_{0}}\right)$ such that $\varphi$ normalises $H_{Q_{0}}^{\mathcal{R}_{Q_{0}}}$. Let $W:=\left\{w_{0}, w_{1}, \ldots, w_{s-1}\right\}$ be a transversal for $H_{Q_{0}}$ in $H$ such that $Q_{0}^{w_{i}}=Q_{i}$ for each $i$. For a fixed $R_{0} \in \mathcal{R}_{Q_{0}}$, let $V:=\left\{v_{1}, \ldots, v_{t}\right\}$ be a transversal for $H_{R_{0}}$ in $H_{Q_{0}}$. 


\begin{tabular}{|l|l|l|}
\hline & $L$ & $M$ \\
\hline (i) & $T_{\{\alpha, \beta\}}=\mathbb{Z}_{2}^{2}$ & $T_{(\alpha, \beta)}=\mathbb{Z}_{2}$ \\
(ii) & $\operatorname{P\Gamma L}(2,8)$ & $\operatorname{PSL}(2,8)$ \\
(iii) & $\operatorname{A\Gamma L}(1,8)$ & $\operatorname{AGL}(1,8), \mathbb{Z}_{2}^{3}$ \\
(iv) & $\operatorname{AGL}(1,8)$ & $\mathbb{Z}_{2}^{3}$ \\
(v) & $\mathbb{Z}_{2}^{3}$ & $\mathbb{Z}_{2}^{2}, T_{\{\alpha, \beta\}}, T_{(\alpha, \beta)}$ \\
(vi) & $T_{\ell} \cong A_{4} \times \mathbb{Z}_{2}$ & $\mathbb{Z}_{2}^{3}, T_{(\alpha, \beta)}$ \\
(vii) & $A_{4} \times \mathbb{Z}_{2}$ & $A_{4}, \mathbb{Z}_{2}^{3}, T_{\{\alpha, \beta\}}$ \\
(viii) & $A_{4}$ & $T_{\{\alpha, \beta\}}$ \\
(ix) & $A_{4} \times \mathbb{Z}_{2}$ & $\mathbb{Z}_{2}^{3}$ \\
\hline
\end{tabular}

TABLE 2. $T=\operatorname{P\Gamma L}(2,8)$. (The groups $L$ in lines (vi), (vii) and (ix) are conjugate in $T$ but not equal.)

Let $Q_{i}, Q_{j} \in \mathcal{Q}$, and let $k \in\{1, \ldots, t\}$. Define

$$
P\left(Q_{i}, Q_{j}, k\right)=\left(\bigcup_{R \in \mathcal{R}_{Q_{0}}} R^{w_{i}} \times_{\text {graph }} R^{v_{k} \varphi w_{j}}\right) \subset Q_{i} \times_{\text {graph }} Q_{j}
$$

and let $\mathcal{P}(\mathcal{T}, \mathcal{R}, \varphi)$ denote the set of all $P\left(Q_{i}, Q_{j}, k\right)$ for all $0 \leq i, j \leq s-1,1 \leq k \leq t$.

\section{2-TRANSITIVE EDGE-DECOMPOSITIONS OF $K_{m}$}

Table 3 gives a rough summary of the classification in [15, Theorem 6] of all $T$-transitive edge decompositions $\mathcal{T}=\left(K_{m}, \mathcal{Q}\right)$ where $T$ is a 2-transitive non-abelian simple group. (We examine the case with $T \cong \operatorname{P\Gamma L}(2,8)$ of degree 28 in Section 2.2.) Sibley's classification draws on and extends classifications of a number of closely related structures, including linear spaces (see Lemma 2.3) and also 1-factorisations of $K_{m}$. (A 1-factorisation of $K_{m}$ is a partition $\mathcal{F}$ of the edge set such that for each $F \in \mathcal{F}$, the subgraph of $K_{m}$ induced by $F$ has valency 1 and is incident with every vertex of $K_{m}$. The 1 -factorisations of $K_{m}$ preserved by a 2-transitive group were classified in [4].) In Table 3 we refer to some of these connections, and also to Constructions 2.1 and 2.2 which are paraphrased from $[15]$.

The numbering of the cases in Table 3 corresponds to the numbering of the Examples in [15]; so for a more detailed description of Case $n$, see Example $n$ of [15].

Construction 2.1. (see [15, Example 5]) Let $T=\operatorname{PSL}(a, 2)$ and let $K_{m}$ be the complete graph with vertex set $\mathrm{PG}(a-1,2)$. For each $\gamma \in V K_{m}$, let $Q(\gamma)$ be the set of all edges $\{\alpha, \beta\}$ of $K_{m}$ such that $\alpha, \beta$ and $\gamma$ are co-linear in $\operatorname{PG}(a-1,2)$ and $\gamma \neq \alpha$ or $\beta$. Let $\mathcal{Q}=\left\{Q(\gamma) \mid \gamma \in V K_{m}\right\}$.

Construction 2.2. (see [15, Examples 6,7 and 8]) Let $T \leq \operatorname{PSL}(a, q)$ and let $K_{m}$ be the complete graph with vertex set $\mathrm{PG}(a-1,2)$. Let $\mathcal{Q}^{\prime}$ be the partition of $A K_{m}$ corresponding to the line set of PG(a-1,3) (see Lemma 2.3), and assume that for each $Q^{\prime} \in \mathcal{Q}^{\prime}$, the (complete) subgraph of $K_{m}$ corresponding to $Q^{\prime}$ admits a $T_{Q^{\prime}}$-invariant 1-factorisation $\mathcal{F}_{Q^{\prime}}$. Let $\mathcal{Q}=\bigcup_{Q^{\prime} \in \mathcal{Q}^{\prime}} \mathcal{F}_{Q^{\prime}}$.

In order to prove Theorem 1.2 we need to give some more detailed information about certain classes of almost simple 2-transitive decompositions of $K_{m}$.

2.1. 2-transitive decompositions corresponding to 2-transitive linear spaces. A linear space $\mathcal{D}$ is a set $\mathcal{V}$ of points together with a set $\mathcal{L}$ of lines (subsets of points) such that each pair of points lies in exactly one line. The automorphism group of $\mathcal{D}$, denoted by 


\begin{tabular}{|c|l|l|l|}
\hline Case & $T$ & $m$ & Description of $\mathcal{Q}$ \\
\hline 1 & - & - & Each part in $\mathcal{Q}$ contains exactly one edge. \\
2 & $\operatorname{PSL}(a, q)$ & $\sum_{i=0}^{a} q^{i}$ & Constructed from a linear space (see Lemma 2.3). \\
& $\operatorname{PSU}(3, q)$ & $q^{3}+1$ & \\
& ${ }^{2} G_{2}(q)$ & $q^{3}+1$ & \\
3 & $A_{7}$ & 15 & \\
& $\operatorname{PSL}(2, q)$ & $q+1$ & 1-factorisation (see $[4])$. \\
5 & $q=5,7$ or 11 & & \\
6 & $\operatorname{PSL}(a, 2)$ & $\sum_{i=0}^{a} 2^{i}$ & Construction 2.1 \\
7 & $\operatorname{PSL}(a, 3)$ & $\sum_{i=0}^{a} 3^{i}$ & Construction 2.2 \\
8 & $\operatorname{PSU}(3,5)$ & $\sum_{i=0}^{a} 5^{i}$ & Construction 2.2 \\
& $q=3$ or 5 & $q^{3}+1$ & Construction 2.2 \\
9 & $\operatorname{Sp}(2 l, 2)$ & $2^{2 l-1} \pm 2^{l-1}$ & See Section 2.3. \\
10 & $\operatorname{PSU}(3,3)$ & 28 & Each part in $\mathcal{Q}$ consists of 6 vertex-disjoint edges. \\
11 & $\operatorname{PSL}(2,9)$ & 10 & Each part in $\mathcal{Q}$ consists of 3 vertex-disjoint edges. \\
\hline
\end{tabular}

TABLE 3. The $T$-transitive edge-decompositions where $T$ is a non-abelian simple 2-transitive group.

AutD , is the group of all permutations of $\mathcal{V}$ which preserve $\mathcal{L}$, and $\mathcal{D}$ is called 2-transitive if Aut $\mathcal{D}$ is 2-transitive on $\mathcal{V}$. Every 2-transitive linear space corresponds to a 2-transitive decomposition of a complete graph into complete subgraphs. This correspondence is given in the following lemma (which is essentially a special case of [14, Lemma 2.1] concerning partial linear spaces). Given a graph $\Gamma$ and a partition $\mathcal{P}$ of $A \Gamma$, for each $P \in \mathcal{P}$ we write $\Gamma_{P}$ for the subgraph of $\Gamma$ with $A \Gamma_{P}=P$ and $V \Gamma_{P}$ the set of all vertices incident with arcs in $P$.

\section{Lemma 2.3.}

(i) Let $\mathcal{D}:=(\mathcal{V}, \mathcal{L})$ be a 2-transitive linear space, and suppose that $G$ is a 2-transitive subgroup of AutD. Let $\Gamma$ be the complete graph with vertex set $\mathcal{V}$. For each $\ell \in \mathcal{L}$, let $P_{\ell}$ be the set of all unordered pairs of distinct elements of $\ell$, and let $\mathcal{P}=\left\{P_{\ell} \mid \ell \in \mathcal{L}\right\}$. Then $(\Gamma, \mathcal{P})$ is a $G$-transitive decomposition, and each $\Gamma_{P_{\ell}}$ is a complete subgraph of $\Gamma$.

(ii) Let $(\Gamma, \mathcal{P})$ be a $G$-transitive decomposition where $G$ is 2-transitive and $\Gamma$ is a complete graph such that for each $P \in \mathcal{P}$, the subgraph $\Gamma_{P}$ is a complete subgraph of $\Gamma$. Let $\mathcal{V}=V \Gamma$, and let $\mathcal{L}=\left\{V \Gamma_{P} \mid P \in \mathcal{P}\right\}$. Then $G$ is a 2-transitive subgroup of AutD and hence $(\mathcal{V}, \mathcal{L})$ is a 2-transitive linear space.

The 2-transitive linear spaces were classified in [9]. Theorem 2.4 lists those preserved by a 2 -transitive almost simple group.

Theorem 2.4 (Kantor). Let $\mathcal{D}$ be a linear space and suppose that $T \leq$ AutD where $T$ is the socle of a 2-transitive almost simple group. Then one of the following holds

(i) $T=\operatorname{PSL}(a, q)$ where $a \geq 3$ and $\mathcal{D}=\operatorname{PG}(a-1, q)$

(ii) $T=\operatorname{PSU}(3, q)$ with $q \geq 3$ and $\mathcal{D}$ is an Hermitian unital. That is, for a 3dimensional vector space $V$ over $\operatorname{GF}\left(q^{2}\right)$ with a non-degenerate Hermitian form, 
the points of $\mathcal{D}$ are the totally isotropic 1-subspaces of $V$, and each line is the set of points contained in a non-degenerate 2-space.

(iii) $T={ }^{2} G_{2}(q)$ and $\mathcal{D}$ is the same linear space as in (ii).

(iv) $T=A_{7}$ and $\mathcal{D}=\operatorname{PG}(3,2)$.

We now give a lemma concerning line stabilisers for almost simple 2-transitive linear spaces.

Lemma 2.5. Suppose that $\mathcal{D}$ is a linear space and $T$ is a non-abelian simple 2-transitive subgroup of AutD. Then for any line $\ell$ of $\mathcal{D}$, either

(a) the permutation group induced on $\ell$ by $T_{\ell}$ is $\operatorname{PGL}(2, q)$, or

(b) we are in case (iii) of Theorem 2.4 and the permutation group induced on $\ell$ by $T_{\ell}$ contains $\operatorname{PSL}(2, q)$, and if $q=3$ it is equal to $\operatorname{PSL}(2, q) \cong A_{4}$.

Proof. We consider each of the cases in Theorem 2.4. In case (i) the linear space is $\operatorname{PG}(a-1, q)$, with $T=\operatorname{PSL}(a, q)$. The points of $\mathcal{D}$ are the 1 -spaces of an $a$-dimensional vector space $V$ over $\operatorname{GF}(q)$, and each line is the set of 1 -spaces contained in some 2-space of $V$. Hence the induced action of $T_{\ell}$ on $\ell$ is that of $\operatorname{PGL}(2, q)$.

In Case (ii) the result follows from [12, Proof of Lemma 2.8]. (More details can be found in [7, p. 132].)

If we are in case (iii) of Theorem 2.4, then $T={ }^{2} G_{2}(q)$ and according to the proof of Theorem 1 in [9], $T_{\ell}^{\ell}$ contains $\operatorname{PSL}(2, q)$ acting 2-transitively on $\ell$, and is equal to $\operatorname{PSL}(2,3) \cong A_{4}$ if $q=3$.

In case (iv) we have $T_{\ell}^{\ell}=\operatorname{PGL}(2,2)$.

2.2. 2-transitive decompositions preserved by $\operatorname{P\Gamma L}(2,8)$ of degree 28. In [15], Sibley identifies and describes most of the $T$-transitive decompositions $\left(K_{28}, \mathcal{Q}\right)$ where $T=\mathrm{P} \Gamma \mathrm{L}(2,8)$ of degree 28 . In recomputing these decompositions we discovered a further three examples that had been overlooked in [15, Theorem 7]. We give here the complete classification. The existence of these decompositions was discovered through computation with Magma, and we refer to Magma computations in the proof of Theorem 2.6.

Theorem 2.6. Let $T=\operatorname{P\Gamma L}(2,8)$ of degree 28 , and suppose that $\left(K_{28}, \mathcal{Q}\right)$ is a (possibly degenerate) T-transitive decomposition. Let $\{\alpha, \beta\} \in E K_{28}$, and let $Q \in \mathcal{Q}$ be the part containing $\{\alpha, \beta\}$. Then the stabiliser $T_{Q}$ appears in Table 4.

\begin{tabular}{|l|l|l|}
\hline & $T_{Q}$ & $Q$ \\
\hline (i) & $T:=\operatorname{P\Gamma L}(2,8)$ & $K_{28}$ \\
(ii) & $T_{\{\alpha, \beta\}} \times \mathbb{Z}_{2}$ & $\{\alpha, \beta\}$ \\
(iii) & $T_{\ell} \cong A_{4} \times \mathbb{Z}_{4}$ \\
(iv) & $\mathrm{AGL}(1,8)$ & $K_{4}$ \\
(v) & $\mathrm{PSL}(2,8)$ & -factor of $K_{28}$ \\
(vi) & $S \cong \mathbb{Z}_{2}^{3}$, the 8 translations from AGL $(1,8)$ & 9-factor of $K_{28}$ \\
(vii) & $\mathrm{A} \Gamma \mathrm{d}(1,8)$ & disjoint edges \\
(viii) & $C_{1} \cong A_{4} \times \mathbb{Z}_{2}$ & -factor of $K_{28}$ \\
(ix) & $D \cong A_{4}\left(D \leq C_{1}\right)$ & 3 disjoint edges \\
(x) & $C_{2} \cong A_{4} \times \mathbb{Z}_{2}$ & 6 disjoint edges \\
\hline
\end{tabular}

TABLE 4. Transitive decompositions preserved by $\mathrm{P} \Gamma L(2,8)$ of degree 28.

Remark. Lines (i)-(vii) of Table 4 are numbered to correspond with [15, Theorem 7], while lines (viii)-(x) contain new examples. (Note that in the proof of [15, Theorem 7] on p 131, $\operatorname{AGL}(2,8)$ and $\operatorname{A\Gamma L}(2,8)$ should read $\operatorname{AGL}(1,8)$ and $\operatorname{A\Gamma L}(1,8)$ respectively.) 
Proof. Lines (i)-(vii) of Table 4 correspond to possibilities (i)-(vii) of [15, Theorem 7]. We now explain how lines (viii)-(x) arise.

By [9], $T$ preserves a $(28,4,1)$ linear space $\mathcal{D}=(\mathcal{V}, \mathcal{L})$. Let $\ell \in \mathcal{L}$ be the unique line of $\mathcal{D}$ containing the points $\alpha, \beta$. Then $T_{\{\alpha, \beta\}} \leq T_{\ell}$, and hence $T_{\ell}$ yields a $T$-transitive decomposition $\left(K_{28}, \mathcal{Q}\right)$ where $V K_{28}=\mathcal{V}$ and where $\mathcal{Q}=\left(\{\alpha, \beta\}^{T_{\ell}}\right)^{T}$ (line (iii) of Table 4). By Lemma 2.5 (b), $T_{\ell}^{\ell}$ is permutationally isomorphic to $A_{4}$. Since $T_{\ell}$ has order 24 , it follows that the kernel $K$ of the action of $T_{\ell}$ on $\ell$ is isomorphic to $\mathbb{Z}_{2}$, and that $T_{\ell}$ has a unique Sylow 2-subgroup $S$ containing $K$. Moreover since $\left|T: T_{\ell}\right|=63$ is odd, $S$ is a Sylow 2-subgroup of $T$ and hence $S \cong \mathbb{Z}_{2}^{3}$. Thus $T_{\ell} \cong \mathbb{Z}_{2}^{3} \rtimes \mathbb{Z}_{3}=K \times\left(\mathbb{Z}_{2}^{2} \rtimes \mathbb{Z}_{3}\right)$. Since $T_{\ell} / K \cong A_{4}$ it follows that $T_{\ell} \cong K \times A$ where $A \cong A_{4}$. Then we have $T_{\{\alpha, \beta\}}=K \times(A)_{\{\alpha, \beta\}} \cong \mathbb{Z}_{2} \times \mathbb{Z}_{2}$. There is exactly one proper subgroup of $A$ containing $(A)_{\{\alpha, \beta\}}$, namely $S \cap A \cong \mathbb{Z}_{2}^{2}$, and hence the only subgroup of $T_{\ell}$ containing $T_{\{\alpha, \beta\}}$ is the one in line (vi) of Table 4.

We used Magma to determine the following information:

(a) $T_{\ell}$ has three orbits on $V K_{28}$. These are $\ell$, which has 4 points; and two orbits $O_{1}$ and $\mathrm{O}_{2}$ each of length 12 .

(b) $T_{\ell}^{O_{i}}$ is non-regular for each $i$.

(c) $T_{\ell}^{O_{1}}$ is not permutationally isomorphic to $T_{\ell}^{O_{2}}$.

Thus, for each $i, T_{\ell}^{O_{i}}$ may be represented by the coset action of $T_{\ell}$ on some core-free subgroup $L_{i}$ of index 12 in $T_{\ell}$ (so in other words $L \neq K$ ). Let $\tau$ be an involution in $A$, and let $\sigma$ be the generator of $K$. Then $L_{i}$ is conjugate in $T_{\ell}$ to either $\langle\tau\rangle$ or $\langle\tau \sigma\rangle$ (and $L_{1}$ is not conjugate to $L_{2}$ ). Assume without loss of generality that $L_{1}=\langle\tau\rangle$ and $L_{2}=\langle\tau \sigma\rangle$. We will show that for each $i$, there exist $\gamma_{i}, \delta_{i} \in O_{i}$ such that $T_{\left\{\gamma_{i}, \delta_{i}\right\}} \leq T_{\ell}$.

Let $\psi_{i}:\left[T_{\ell}: L_{i}\right] \longrightarrow O_{i}$ be the bijection defining the permutational equivalence between the action of $T_{\ell}$ on $\left[T_{\ell}: L_{i}\right]$ and on $O_{i}$. Let $\tau^{\prime} \neq \tau$ be an involution in $A$, and let $\psi_{1}(\langle\tau\rangle)=$ $\gamma_{1}$ and $\psi_{1}\left(\langle\tau\rangle \tau^{\prime}\right)=\delta_{1}$. Then the stabiliser in $T_{\ell}$ of $\left\{\gamma_{1}, \delta_{1}\right\}$ is equal to $\left\langle\tau, \tau^{\prime}\right\rangle \cong \mathbb{Z}_{2}^{2}$. Since $\left|T_{\left\{\gamma_{1}, \delta_{1}\right\}}\right|=4$ it follows that $T_{\left\{\gamma_{1}, \delta_{1}\right\}}=\left\langle\tau, \tau^{\prime}\right\rangle \leq T_{\ell}$. On the other hand let $\psi_{2}(\langle\tau \sigma\rangle)=\gamma_{2}$ and $\psi_{2}(\langle\tau \sigma\rangle \sigma)=\delta_{2}$. Then the stabiliser in $T_{\ell}$ of $\left\{\gamma_{2}, \delta_{2}\right\}$ is equal to $\langle\tau \sigma, \sigma\rangle \cong \mathbb{Z}_{2}^{2}$. Since $\left|T_{\left\{\gamma_{2}, \delta_{2}\right\}}\right|=4$ it follows that $T_{\left\{\gamma_{2}, \delta_{2}\right\}}=\langle\tau \sigma, \sigma\rangle \leq T_{\ell}$.

In each case, the index of $T_{\left\{\gamma_{i}, \delta_{i}\right\}}$ in $T_{\ell}$ is 6 . Since $\left|O_{i}\right|=12$, it follows that $\left\{\gamma_{i}, \delta_{i}\right\}^{T_{\ell}}$ consists of 6 disjoint pairs.

Now, observe that the stabiliser $\left(T_{\ell}\right)_{\left\{\gamma_{1}, \delta_{1}\right\}}=\left\langle\tau, \tau^{\prime}\right\rangle$ is contained in the subgroup $A$ of $T_{\ell}$. The index of $\left(T_{\ell}\right)_{\left\{\gamma_{1}, \delta_{1}\right\}}$ in $A$ is 3 , and since $\left\{\gamma_{1}, \delta_{1}\right\}^{A} \subset\left\{\gamma_{1}, \delta_{1}\right\}^{T_{\ell}}$, it follows that the orbit $\left\{\gamma_{1}, \delta_{1}\right\}^{A}$ consists of 3 disjoint pairs.

Now, since $T$ acts transitively on ordered pairs of points, there exist elements $t_{1}, t_{2} \in T$ with $\left\{\gamma_{i}, \delta_{i}\right\}^{t_{i}}=\{\alpha, \beta\}$. Writing $C_{i}:=T_{\ell}^{t_{i}}$ and $D:=A^{t_{1}}$, we have $T_{\{\alpha, \beta\}}<C_{i}<T$, and $T_{\{\alpha, \beta\}}<D<T$, where $\{\alpha, \beta\}^{C_{i}}$ consists of 6 disjoint edges and $\{\alpha, \beta\}^{D}$ consists of 3 disjoint edges. This gives lines (viii)-(x) of Table 4 .

2.3. 2-transitive decompositions for $\operatorname{Sp}(2 l, 2)$. In this section we give some results pertaining to the 2-transitive actions of $\operatorname{Sp}(2 l, 2)$, in preparation for the proof of Theorem 1.2. We first explain the notation used in [8, Section 7.7] to describe these actions of $\operatorname{Sp}(2 l, 2)$.

Let $T=\operatorname{Sp}(2 l, 2)$ with $l \geq 3$ and let $V$ be a $2 l$-dimensional vector space over $\operatorname{GF}(2)$. Let

$$
e=\left(\begin{array}{ll}
\mathbf{0} & \mathbf{I} \\
\mathbf{0} & \mathbf{0}
\end{array}\right) \text { and } f=\left(\begin{array}{ll}
\mathbf{0} & \mathbf{I} \\
\mathbf{I} & \mathbf{0}
\end{array}\right)
$$

where $\mathbf{0}$ and $\mathbf{I}$ denote the $l \times l$ zero and identity matrices respectively. Define a symmetric bilinear form $\phi$ by $\phi(u, v):=u f v^{\top}$ and for each $c \in V$ define $\theta_{a}: V \longrightarrow \operatorname{GF}(2)$ by $\theta_{a}(u)=u e u^{\top}+u f a^{\top}$. (Note that [8] uses $\varphi$ to denote the form $\phi$; however this conflicts 
with our usage of $\varphi$ in Construction 1.3.) For each $c \in V$, define a transvection $t_{c}$ by $t_{c}: u \longmapsto u+\phi(u, c) c$. Then $t_{c} \in \operatorname{Sp}(2 l, 2)$ and $x^{-1} t_{a} x=t_{a x}$ for all $x \in \operatorname{Sp}(2 l, 2)$. For each $a$ and $c$ and $u \in V$ we have $\theta_{a}^{t_{c}}(u)=\theta_{a}\left(u t_{c}^{-1}\right)$. This leads to the following result, which is taken directly from [8].

Lemma 2.7. $\quad$ (i) For all $a, c \in V$ we have

$$
\theta_{a}^{t_{c}}= \begin{cases}\theta_{a} & \text { if } \theta_{a}(c)=1 \\ \theta_{a+c} & \text { if } \theta_{a}(c)=0\end{cases}
$$

(ii) For all $a, b \in V$ there is at most one $c \in V$ such that $t_{c}$ maps $\theta_{a}$ onto $\theta_{b}$. Such $a$ $c$ exists if and only if $\theta_{0}(a)=\theta_{0}(b)$ (and then $c=a+b$ ).

The group $\operatorname{Sp}(a, 2)=\left\langle t_{c} \mid c \in V\right\rangle$, and has two orbits on the set $\left\{\theta_{a} \mid a \in V\right\}$. These orbits are

$$
\Omega^{+}:=\left\{\theta_{a} \mid \theta_{0}(a)=0\right\} \quad \text { and } \quad \Omega^{-}:=\left\{\theta_{a} \mid \theta_{0}(a)=1\right\} .
$$

It is shown in $[8$, Theorem $7.7 \mathrm{~A}]$ that $\operatorname{Sp}(2 l, 2)$ acts 2 -transitively on each of $\Omega^{+}$and $\Omega^{-}$for each $l \geq 2$.

The proof of Theorem 1.2 in the case $T=\operatorname{Sp}(2 l, 2)$ of degree $2^{2 l-1} \pm 2^{l-1}$ involves several key steps which we prove here as separate lemmas. First we give an explanation of the family of transitive decompositions in [15, Example 9].

Let $\Omega^{\prime}$ equal either $\Omega^{+}$or $\Omega^{-}$, and let $K_{m}$ be the complete graph with vertex set $\Omega^{\prime}$. The $T$-transitive decomposition $\left(K_{m}, \mathcal{Q}\right)$ in [15, Example 9] is such that for an edge $\left\{\theta_{a}, \theta_{b}\right\}$, the part of $\mathcal{Q}$ containing $\left\{\theta_{a}, \theta_{b}\right\}$ is the set of all edges $\left\{\theta_{c}, \theta_{d}\right\}$ such that $c+d=a+b$. For each vector $v$ in $V \backslash\{0\}$, define $Q_{v}$ to be the part in $\mathcal{Q}$ (if one exists) consisting of all edges $\left\{\theta_{a}, \theta_{b}\right\}$ with $a+b=v$.

Lemma 2.8. Let $t_{c}$ and $t_{d}$ be transvections in $T$. Then $t_{c}=t_{d}$ if and only if $c=d$.

Lemma 2.9. Let $v, c \in V \backslash\{0\}$. Then $t_{c}$ fixes $Q_{v}$ setwise if and only if $t_{c}$ fixes $v$ (in the action of $T$ on $V \backslash\{0\})$.

Proof. Assume first that $t_{c}$ fixes $v$, and let $a, b \in V$ be such that $v=a+b$. Then $v=v^{t_{c}}=$ $v+\phi(v, c) c$, which implies that $\phi(v, c)=0$. This means that $\phi(a+b, c)=\phi(a, c)+\phi(b, c)=$ 0 and hence that $\phi(c, a)=\phi(c, b)$. Now we have $\theta_{a}(c)=c e c^{\top}+c f a^{\top}=\theta_{0}(c)+\phi(c, a)$ and $\theta_{b}(c)=\theta_{0}(c)+\phi(c, b)$. Hence, since $\phi(c, a)=\phi(c, b)$, either $\theta_{a}(c)=0$ and $\theta_{b}(c)=0$, or $\theta_{a}(c)=1$ and $\theta_{b}(c)=1$. It follows from Lemma 2.7 that $\left\{\theta_{a}, \theta_{b}\right\}^{t_{c}}$ equals either $\left\{\theta_{a}, \theta_{b}\right\}$ or $\left\{\theta_{a+c}, \theta_{b+c}\right\}$, both of which are contained in $Q_{v}$. So the transvection $t_{c}$ fixes $Q_{v}$ setwise. Conversely, suppose that $t_{c}$ fixes $Q_{v}$ setwise. Then $\left\{\theta_{a}, \theta_{b}\right\}^{t_{c}}=\left\{\theta_{a+d}, \theta_{b+d}\right\}$ for some $d$. Lemma 2.7 implies that $d$ is either 0 or $c$, and that, in either case, $\theta_{a}(c)=\theta_{b}(c)$. This means that $\theta_{0}(c)+\phi(a, c)=\theta_{0}(c)+\phi(b, c)$ and hence that $0=\phi(a, c)+\phi(b, c)=\phi(v, c)$. Hence $t_{c}$ fixes $v$.

Lemma 2.10. $T_{Q_{v}}=T_{v}$.

Proof. Let $S_{v}$ denote the set of all transvections in $T$ fixing $v$ and let $B$ denote the set of vectors in $V$ fixed by every transvection in $S_{v}$. Recall that for any transvection $t_{c}$ and any $x \in T$ we have $t_{c}^{x}=t_{c x}$. From this it follows that $T$ acts transitively by conjugation on the set of all non-trivial transvections. Since $S_{v}^{x}=S_{v x}$ for any $x \in T$, we find that $\left|S_{u}\right|=\left|S_{w}\right|$ for all $u, w \in V$. Suppose that $u, w \in B$. Then by the definition of $B$, each element of $S_{v}$ fixes both $u$ and $w$; so $S_{v} \subseteq S_{u} \cap S_{w}$. Hence $S_{u}=S_{w}=S_{v}$. If for some $x \in T$ we have $u^{x} \notin B$, then $S_{u}^{x} \neq S_{v}=S_{u}$ and so $S_{w}^{x} \neq S_{w}$. This means that $w^{x} \notin B$, which implies that $B^{x} \cap B=\varnothing$ and hence that $B$ is a block of imprimitivity for $T$. But $T$ acts primitively on $V \backslash\{0\}$, and so $B$ must be $\{v\}$ (since no non-trivial transvection fixes every vector in $V \backslash\{0\})$. Now, by Lemma 2.9, $T_{Q_{v}}$ contains $S_{v}$ and no other transvections. 
Hence for any $x \in T_{Q_{v}}$ we have $S_{v}^{x}=S_{v^{x}}=S_{v}$. So $x$ must fix $v$, and hence $T_{Q_{v}} \leq T_{v}$. On the other hand, given that each part $Q \in \mathcal{Q}$ corresponds to a unique vector $v \in V \backslash\{0\}$, the size of $\mathcal{Q}$ is at most $|V|-1$. Hence the index of $T_{Q_{v}}$ in $T$ cannot exceed $|V|-1$, and so $T_{Q_{v}}=T_{v}$.

Now we describe the structure of the group $T_{v}$. Although this information is wellknown in the theory of classical groups, it does not appear to be covered explicitly in a convenient reference. We outline a proof of Lemma 2.11, omitting routine computations, and we acknowledge unpublished lecture notes by David Vogan for the notation and method of proof.

Since the form $\phi$ is non-degenerate and $v$ is non-zero, we may choose a vector $u \in V$ with $\phi(v, u)=1$. Let $W=\{w \in V \mid \phi(v, w)=\phi(u, w)=0\}$. Then $W$ is a (2l-2)-dimensional subspace of $V$ and $V=\langle v, u\rangle \oplus W$. We define three types of linear transformations of $V$ by specifying their actions on $v, u$ and $W$. For $x \in G F(2), w_{1} \in W$ and $g \in S p(W)$, define maps $z_{x}, n_{w_{1}}$ and $s_{g}$, each from $V$ to $V$, by

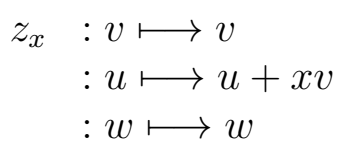

$$
\begin{aligned}
n_{w_{1}} & : v \longmapsto v \\
& : u \longmapsto u+w_{1} \\
& : w \longmapsto w+\phi\left(w_{1}, w\right) v
\end{aligned}
$$$$
\begin{aligned}
s_{g} & : v \longmapsto v \\
& : u \longmapsto u \\
& : w \longmapsto w^{g}
\end{aligned}
$$

for all $w \in W$. It is easily verified that each such linear transformation preserves $\phi$ and hence is contained in $T_{v}$.

Lemma 2.11. $T_{v}$ has normal subgroups $Z$ and $N$ where $Z<N,|Z|=2$, and $N / Z \cong$ $\mathbb{Z}_{2}^{2 l-2}$. Furthermore, $T_{v}$ has a subgroup $P$ isomorphic to $S p(2 l-2,2)$, such that $T_{v} / Z=$ $N / Z \rtimes P Z / Z \cong \mathbb{Z}_{2}^{2 l-2} \cdot \operatorname{Sp}(2 l-2,2)$. In particular, $N / Z$ is the unique minimal normal subgroup of $T_{v} / Z$.

Proof. Let $z_{x}, n_{w_{1}}$ and $s_{g}$ be as defined above. Then it is routine to verify that the sets $Z=\left\{z_{x} \mid x \in G F(2)\right\}$ and $N=\left\{z_{x} n_{w_{1}} \mid w_{1} \in W, x \in G F(2)\right\}$ are subgroups of $T_{v}$, and that $Z<N$ and $|Z|=2$. Also, we have that $Z \triangleleft N$ with $N / Z \cong W \cong \mathbb{Z}_{2}^{2 l-2}$. Furthermore, $P=\left\{s_{g} \mid g \in S p(W)\right\} \cong S p(W)$ is a subgroup of $T_{v}$ which normalises $N$ and $Z$, with $\left(z_{x} n_{w_{1}}\right)^{s_{g}}=n_{w_{1}^{g}} z_{x}$ for all $z_{x} n_{w_{1}} \in N$ and $s_{g} \in P$. Using this fact together with the orders of $T_{v}, P$ and $N$, and the fact that $N \cap P$ is trivial, we deduce that $T_{v}=N \rtimes P$, whence we obtain the result.

To prove the next result, we note that the binary operation of $N$ is given by

$$
\left(z_{x_{1}} n_{w_{1}}\right)\left(z_{x_{2}} n_{w_{2}}\right)=z_{x_{1}+x_{2}+\phi\left(w_{1}, w_{2}\right)} n_{w_{1}+w_{2}} .
$$

Lemma 2.12. Let $T_{v}, N$ and $Z$ be as in Lemma 2.11, and suppose that $K \leq N$ with $|N: K|=2$ and $K \triangleleft T_{v}$. Then $Z \leq K$.

Proof. Let $\psi$ denote the homomorphism $K \longrightarrow W: n_{w} z_{x} \longmapsto w$. By Lemma 2.11, ker $\psi$ is either trivial or $Z$. In the latter case $Z \leq K$ as required, so assume that ker $\psi$ is trivial. Then $\psi(K)=W$ since $|N: K|=2$. Now recall that $W$ is a vector space over $\mathrm{GF}(2)$, and fix $i \in \mathrm{GF}(2)$. Suppose that for all $n_{w} z_{x} \in K$ with $w$ non-trivial we have $x=i$. There exist $w_{1}, w_{2} \in W$ with $w_{1} \neq w_{2}$ and $\phi\left(w_{1}, w_{2}\right)=i-1$, and so $\left(n_{w_{1}} z_{i}\right)\left(n_{w_{2}} z_{i}\right)=n_{w_{1}+w_{2}} z_{i+i+i-1}=n_{w_{1}+w_{2}} z_{i-1}$. That is to say, $K$ contains a non-trivial element $n_{w_{1}+w_{2}} z_{x}$ with $x \neq i$ which is a contradiction; hence there exist elements $n_{w_{1}} z_{0}$ and $n_{w_{2}} z_{1}$ in $K$. Now $P$ acts transitively as the symplectic group on $W$, and so there exists $s_{g} \in P$ with $w_{1}^{g}=w_{2}$. Since $K \triangleleft T_{v}$, the group $P$ normalises $K$, and so we have $\left(n_{w_{1}} z_{0}\right)^{s_{g}}=n_{w_{2}} z_{0} \in K$. But then $n_{w_{2}} z_{0} n_{w_{2}} z_{1}=n_{w_{2}+w_{2}} z_{0+1+\phi\left(w_{2}, w_{2}\right)}=z_{1} \in K$. Thus $Z=\left\langle z_{1}\right\rangle \leq K$ which contradicts the assumption that $\operatorname{ker} \psi$ is trivial. Hence $Z \leq K$. 


\section{Proof of Theorem 1.2.}

First we give a lemma which essentially proves part (i) of Theorem 1.2.

Lemma 3.1. Let $G, \Gamma, \mathcal{P}(\mathcal{T}, \mathcal{R}, \varphi)$ and $\mathcal{Q}$ be as in Notation 1.1 , and let $(\alpha, \beta)$ be an arc in $Q_{0} \in \mathcal{Q}$. Let $R_{0}$ be the part in $\mathcal{R}$ containing $(\alpha, \beta)$, and let $L:=T_{Q_{0}}$ and $M:=T_{R_{0}}$. Then $T_{(\alpha, \beta)} \leq M \triangleleft L \leq T$ and $T_{\{\alpha, \beta\}} \leq L$; and we have $\mathcal{Q}=Q_{0}^{T}$ with $Q_{0}=(\alpha, \beta)^{L}$, and $\mathcal{R}=R_{0}^{T}$ with $R_{0}=(\alpha, \beta)^{M}$. Moreover, the homomorphism $\varphi$ is determined by an automorphism $\varphi_{0}$ of $L / M$.

Proof. Note that $T$ is 2-transitive on $\Omega_{0}$, so both $\mathcal{Q}$ and $\mathcal{R}$ are systems of imprimitivity for $T$ in its action on $A \Delta$. Thus $T_{(\alpha, \beta)} \leq M$, and since $\mathcal{Q}$ is symmetric (and therefore essentially an edge-partition) we have $T_{\{\alpha, \beta\}} \leq L$. Since $T$ is 2 -transitive we have $\mathcal{Q}=Q_{0}^{T}$ with $Q_{0}=(\alpha, \beta)^{L}$, and $\mathcal{R}=R_{0}^{T}$ with $R_{0}=(\alpha, \beta)^{M}$.

Now since $\mathcal{R}$ refines $\mathcal{Q}$ and $R_{0} \subset Q_{0}$, we have $M \leq L$. By assumption (see Construction 1.3), $H_{Q_{0}}^{\mathcal{R}_{Q_{0}}}$ is regular, implying that $H_{R_{0}} \triangleleft H_{Q_{0}}$. Now $L=T \cap H_{Q_{0}}$ and $M=T \cap H_{R_{0}}$, and so $M \triangleleft L$. Thus $L / M$ is regular and permutationally isomorphic to $H_{Q_{0}}^{\mathcal{R}_{Q_{0}}}$, and the element $\varphi$ of $N_{\operatorname{Sym}\left(\mathcal{R}_{Q_{0}}\right)}\left(H_{Q_{0}}^{\mathcal{R}_{Q_{0}}}\right)$ may be identified with an element $\varphi_{0}$ of $\operatorname{Aut}(L / M)$.

From Sibley's classification [15] we can determine all possibilities for the subgroup $L$. Note that we need to consider the possibility $L=T$ (in which case the decomposition $\mathcal{Q}$ is degenerate) since as long as $|\mathcal{R}|>1$, the partition $\mathcal{P}(\mathcal{Q}, \mathcal{R}, \varphi)$ will still be non-degenerate.

Before proving Theorem 1.2 we make some further observations about $L$ and $M$. First, if both $L$ and $M$ contain the edge stabiliser $T_{\{\alpha, \beta\}}$, then the transitive decompositions corresponding to $L$ and $M$ are both described in Table 3 (and in greater detail in [15]). If $M=T_{(\alpha, \beta)}$ then the corresponding transitive decomposition is such that each part in the arc partition contains exactly one arc. The only remaining situation has $M$ properly containing $T_{(\alpha, \beta)}$ but not containing $T_{\{\alpha, \beta\}}$. The following lemma shows what happens in this case.

Lemma 3.2. Suppose that $T$ is a 2-transitive group and that $T_{\{\alpha, \beta\}} \leq L \leq T$ with $T_{\{\alpha, \beta\}}$ maximal in L. Suppose also that $M \triangleleft L$ such that $T_{(\alpha, \beta)}<M$ and $T_{\{\alpha, \beta\}} \not \leq M$. Then $|L: M|=2$ and $(\alpha, \beta)^{M}$ is a 'directed copy' of the undirected $(\alpha, \beta)^{L}$; that is, for every pair $(\gamma, \delta),(\delta, \gamma)$ of arcs in $(\alpha, \beta)^{L}$, exactly one of $(\gamma, \delta)$ and $(\delta, \gamma)$ is in $(\alpha, \beta)^{M}$.

Proof. First, observe that $T_{\{\alpha, \beta\}}<\left\langle T_{\{\alpha, \beta\}}, M\right\rangle \leq L$ and so by the maximality of $T_{\{\alpha, \beta\}}$ in $L$, we have $\left\langle T_{\{\alpha, \beta\}}, M\right\rangle=L$. Since $T_{\{\alpha, \beta\}}$ normalises $M$, we have $L=M T_{\{\alpha, \beta\}}$ and hence $T_{\{\alpha, \beta\}} /\left(M \cap T_{\{\alpha, \beta\}}\right) \cong T_{\{\alpha, \beta\}} M / M=L / M$. Since $T_{\{\alpha, \beta\}} \not \leq M$ we have $M \cap T_{\{\alpha, \beta\}}=T_{(\alpha, \beta)}$ and so $|L: M|=\left|T_{\{\alpha, \beta\}}\right| /\left|T_{(\alpha, \beta)}\right|=2$. This implies that $\left|(\alpha, \beta)^{M}\right|=\left|(\alpha, \beta)^{L}\right| / 2$. If $(\alpha, \beta)^{M}$ contained $(\beta, \alpha)$, then $M$ would have to contain an element $x$ swapping $\alpha$ and $\beta$, in which case $\left\langle T_{(\alpha, \beta)}, x\right\rangle=T_{\{\alpha, \beta\}}$ would be a subgroup of $M$, which is not the case. It follows that $(\alpha, \beta)^{M}$ has the form described in the statement.

We need one more lemma before proving Theorem 1.2.

Lemma 3.3. Suppose that $\mathcal{T}=(\Delta, \mathcal{Q})$ is a $T$-transitive decomposition, and let $Q \in \mathcal{Q}$. Let $V \Delta_{Q}$ be the set of all vertices of $\Delta$ incident with arcs in $Q$, and let $\alpha, \beta \in V \Delta_{Q}$. Assume that $T_{(\alpha, \beta)} \leq M \unlhd L \leq T_{Q}$. If $M^{V \Delta_{Q}}=L^{V \Delta_{Q}}$, then $M=L$.

Proof. Since $\alpha, \beta \in V \Delta_{Q}, T_{(\alpha, \beta)}$ contains the kernel $K$ of the action of $T_{Q}$ on $V \Delta_{Q}$. Suppose that $M \neq L$. Then since $M^{V \Delta_{Q}} \cong M / K$ and $L^{V \Delta_{Q}} \cong L / K$, we have $M^{V \Delta_{Q}} \neq$ $L^{V \Delta_{Q}}$, by Lemma 3.2. Hence if $M^{V \Delta_{Q}}=L^{V \Delta_{Q}}$, then $M=L$.

We are now ready to prove Theorem 1.2. 
Proof of Theorem 1.2. Part (i) follows immediately from Lemma 3.1.

We now prove part (ii). For each $L$ with $T_{\{\alpha, \beta\}} \leq L \leq T$ we need to find all $M$ with $T_{(\alpha, \beta)} \leq M \triangleleft L \leq T$. We begin with two observations. The first is that if $T_{(\alpha, \beta)}$ or $T_{\{\alpha, \beta\}}$ were normal in $T$, then $T_{(\alpha, \beta)}$ would be trivial, meaning that $T$ would be sharply 2 -transitive. By [8, p 238], every sharply 2-transitive group is of affine type; hence, since $T$ is almost simple, we cannot have $L=T$ with $M$ equal to either $T_{(\alpha, \beta)}$ or $T_{\{\alpha, \beta\}}$. Second, we note that $T_{(\alpha, \beta)}$ is normal in $T_{\{\alpha, \beta\}}$ for any group $T$, and so we may take $M=T_{(\alpha, \beta)}$ and $L=T_{\{\alpha, \beta\}}$, whence we obtain Line (i) of Table 1 .

We will assume at this point that $T$ is simple (and we will treat the case $T \cong \operatorname{P\Gamma L}(2,8)$ of degree 28 later). Assume also that $T_{(\alpha, \beta)}<M \triangleleft L \leq T$ where $T_{\{\alpha, \beta\}}<L$ (and so $M \neq L$ and $T_{\{\alpha, \beta\}} \neq L$ ). For each 2 -transitive simple group $T$, we will refer to Table 3 to determine all possibilities for $L$. Then for each $L$, either we will show that $M$ and $L$ must occur in some line of Table 1, or we will derive a contradiction (usually with the assumption that $M \neq L$ ).

If $T$ is one of $\operatorname{PSL}(2,11)$ of degree $11, A_{n}$ of degree $n, H S, C_{3},{ }^{2} B_{2}(q)$, or one of the Mathieu groups, then according to [15], $T_{\{\alpha, \beta\}}$ is maximal in $T$, and so $L=T$. Hence $L$ is simple, which contradicts the assumption that $1 \neq M \neq L$.

We will examine the remaining 2-transitive simple groups $T$ in roughly the order in which they appear in [9, Section 2]. For each $T$, we work through the possible cases in Table 3.

CASE $T=\operatorname{PSL}(a, q), m=\left(q^{a}-1\right) /(q-1)$ WITH $a \leq 2, q>3$ : Here $L$ corresponds to a transitive decomposition described in Case 3 or 11 of Table 3. In Case 3, $T$ is one of $\operatorname{PSL}(2,5), \operatorname{PSL}(2,7)$ or $\operatorname{PSL}(2,11)$, and for each of these groups the subgroup $L$ (which is the stabiliser of a 1-factor) is specified in [4] as follows. When $T=\operatorname{PSL}(2,5)$, the subgroup $L$ is permutationally isomorphic to $A_{4}$ acting on the cosets of a subgroup of order 2, which we may assume is $\langle(12)(34)\rangle$. The setwise stabiliser of the two cosets $\langle(12)(34)\rangle$ and $\langle(12)(34)\rangle(13)(24)$ is $V_{4}$ (the Klein 4-group) which is the only proper nontrivial normal subgroup of $A_{4}$, and hence (taking $M \cong V_{4}$ ) we obtain Line (iv) of Table 1. When $T=\operatorname{PSL}(2,7)$, the subgroup $L=S_{4}$ in its action on the cosets of, say, $\langle(123)\rangle$. In this case the stabiliser of an edge is contained in $A_{4}$ (but not in $V_{4}$ ), and hence (taking $\left.M \cong A_{4}\right)$ we obtain Line (iii) of Table 1 . When $T=\operatorname{PSL}(2,11)$, the subgroup $L=A_{5}$ which is simple, and so we have a contradiction with $1 \neq M \neq L$. Now suppose that we are in Case 11. Here $T=\operatorname{PSL}(2,9)$ and $L$ is maximal of order 24; and hence by [5], $L \cong S_{4}$. The order of $T_{(\alpha, \beta)}$ is 4 , and so either $T_{(\alpha, \beta)} \cong \mathbb{Z}_{2}^{2}$ or $T_{(\alpha, \beta)} \cong \mathbb{Z}_{4}$. Assume that $\alpha$ is the 1 -space $\langle(1,0)\rangle$, and let $Z$ denote the centre of $\operatorname{SL}(2,9)$. Let $\omega$ be a primitive element of the multiplicative group of $\mathrm{GF}(9)$, and let

$$
A:=\left(\begin{array}{cc}
\omega & 0 \\
0 & \omega^{-1}
\end{array}\right) \leq \mathrm{SL}(2,9)
$$

Then $X:=\langle Z A\rangle \cong\langle A\rangle /(Z \cap\langle A\rangle)$ is a subgroup of $T_{\alpha}$, and since $|Z \cap\langle A\rangle|=\left|\left\langle A^{4}\right\rangle\right|=2$, it follows that $X$ is cyclic of order 4. Furthermore, since $\left|T_{\alpha}\right|=2^{2} .3^{2}, X$ is a Sylow 2 -subgroup of $T_{\alpha}$. This means that any order 4 subgroup of $T_{\alpha}$ is cyclic, and since $T_{(\alpha, \beta)} \leq T_{\alpha}$, we have $T_{(\alpha, \beta)} \cong \mathbb{Z}_{4}$. But then since $L \cong S_{4}$, we have $N_{L}\left(T_{(\alpha, \beta)}\right)=L$, implying that $M=L$, which is a contradiction.

CASE $T=\operatorname{PSL}(a, q), m=\left(q^{a}-1\right) /(q-1)$ WITH $a \geq 3$ : Here $L$ corresponds to a transitive decomposition occurring in one of Cases 2, 5,6 or 7 of Table 3.

Suppose we are in Case 2; so $L$ is the stabiliser of the unique line $\ell$ of $\operatorname{PG}(a-1, q)$ containing $\alpha$ and $\beta$. Suppose that $q>3$. Then Lemma 2.5 shows that the group $L^{\ell}$ 
induced by $L$ on $\ell$ is almost simple with a 2 -transitive socle, meaning that $T_{(\alpha, \beta)}^{\ell} \neq 1$. Hence $M^{\ell}$ is a non-trivial normal subgroup of $L^{\ell}$, which means that $M^{\ell}$ is 2-transitive on $\ell$. But then by Lemma 3.3, $M$ must equal $L$, which is a contradiction. Assume now that $q=2$. Then $M^{\ell}$ is a proper normal subgroup of $L^{\ell}=\operatorname{PSL}(2,2) \cong S_{3}$, meaning that we can take either $M^{\ell} \cong A_{3}$ or $M^{\ell}=T_{(\alpha, \beta)}^{\ell}$ (both of which contain $T_{(\alpha, \beta)}^{\ell}$ ); this gives us Line (vi) of Table 1 . Finally, assume that $q=3$. Then $L^{\ell}=\operatorname{PGL}(2,3) \cong S_{4}$, of which the only proper non-trivial normal subgroups are $A_{4}$ and $V_{4}$, neither of which contains a stabiliser in $S_{4}$ of two points. This contradicts the assumption that $T_{(\alpha, \beta)} \leq M$.

Suppose now that we are in Case 5 of Table 3 . Here $T=\operatorname{PSL}(a, 2)$ with $a \geq 3$, which we view as $\operatorname{SL}(a, 2)$ acting on an $a$-dimensional vector space $V$ over $\operatorname{GF}(2)$. The group $L=T_{Q}$ where $Q=Q(\gamma)$ as in Construction 2.1 for some $\gamma \in V \backslash\{0\}$; that is, $Q$ consists of all edges $\{\alpha, \beta\}$ with $\alpha, \beta \neq \gamma$ such that $\alpha$ and $\beta$ lie in a 2-subspace together with $\gamma$. Now $L$ is 2-transitive on the set of lines incident with $\gamma$, and hence it is 2-transitive on the set $\left\{\left\{\alpha^{\prime}, \beta^{\prime}\right\} \mid\left(\alpha^{\prime}, \beta^{\prime}\right) \in Q\right\}$. So $L^{Q}$ has a set $\mathcal{Q}^{\prime}$ of $|Q| / 2$ blocks of imprimitivity of size 2 , namely all pairs of the form $\left\{\left(\alpha^{\prime}, \beta^{\prime}\right),\left(\beta^{\prime}, \alpha^{\prime}\right)\right\}$. Let $R \in \mathcal{R}$ with $R \subset Q$ and $(\alpha, \beta) \in R$. If $(\beta, \alpha) \in R$, then $R$ is a union of blocks in $\mathcal{Q}^{\prime}$, and since $L^{\mathcal{Q}^{\prime}}$ is primitive and $R \neq Q$ we obtain $R=\{(\alpha, \beta),(\beta, \alpha)\}$, implying that $M=T_{R}=T_{\{\alpha, \beta\}}$. But then $M$ is not normal in $L$, which is a contradiction. So assume instead that $(\beta, \alpha) \notin R$, and suppose that $|R|>1$. Then $R$ contains $\left(\alpha^{\prime}, \beta^{\prime}\right)$ where $\alpha^{\prime}, \beta^{\prime}$ lie together in a 2-space with $\gamma$ and $\left\{\alpha^{\prime}, \beta^{\prime}\right\} \cap\{\alpha, \beta\}=\varnothing$. Now $T_{\gamma \alpha}$ fixes the arc $(\alpha, \beta)$ and contains an element swapping $\alpha^{\prime}$ and $\beta^{\prime}$. Hence $R$ must contain both $\left(\alpha^{\prime}, \beta^{\prime}\right)$ and $\left(\beta^{\prime}, \alpha^{\prime}\right)$, and it follows that $R$ contains $(\beta, \alpha)$, which is a contradiction. Hence $R=\{(\alpha, \beta)\}$, implying that $M=T_{(\alpha, \beta)}$, which is also a contradiction since $T_{(\alpha, \beta)}$ is not normal in $L$.

We next examine Cases 7 and 6 of Table 3. In each of these cases, the transitive decomposition refines a decomposition corresponding to a 2 -transitive linear space $\mathcal{D}$, and we have

$$
T_{(\alpha, \beta)}^{\ell} \leq M^{\ell} \unlhd L^{\ell} \leq T_{\ell}^{\ell}
$$

where $\ell$ is the line of $\mathcal{D}$ containing $\alpha$ and $\beta$.

Suppose that we are in Case 7 of Table 3. Here $T=\operatorname{PSL}(a, 5)$ and $\mathcal{D}=\operatorname{PG}(a-1,5)$. We know from Lemma 2.5 that $T_{\ell}^{\ell}=\operatorname{PGL}(2,5)$ and the description of Case 7 in [15] that $L^{\ell}$ is the subgroup of $\operatorname{PGL}(2,5)$ fixing a 1-factor of $K_{6}$. By [4], this subgroup is permutationally isomorphic to $S_{4}$ acting on the cosets of, say, $\langle(1234)\rangle$. The stabiliser of two points in this action is generated by a 4-cycle in $S_{4}$, and hence is not contained in any proper normal subgroup of $S_{4}$, and so $M^{\ell}=L^{\ell}$. Lemma 3.3 then implies that $M=L$, which is a contradiction.

Suppose now that we are in Case 6 . Then $T_{\ell}^{\ell}=\operatorname{PGL}(2,3) \cong S_{4}$. As shown in $[15$, Figure 3], $Q:=\{\alpha, \beta\}^{L}$ consists of exactly two disjoint edges. It follows that $T_{\{\alpha, \beta\}}$ has index 2 in $L$, making it a normal subgroup of $L$. Hence if $M=T_{\{\alpha, \beta\}}$ we obtain Line (vii) of Table 1. Now suppose that $\{\gamma, \delta\}$ is the other edge in $Q$. The normal subgroup $M^{\ell}$ of $L^{\ell}$ must contain both $T_{(\alpha, \beta)}^{\ell}$ and its conjugate $T_{(\gamma, \delta)}^{\ell}$. Since $T_{\ell}^{\ell}=S_{4}, T_{(\alpha, \beta)}^{\ell}$ transposes $\gamma$ and $\delta$, and $T_{(\gamma, \delta)}^{\ell}$ transposes $\alpha$ and $\beta$, and so we have $\langle(\alpha \beta),(\gamma \delta)\rangle=T_{\{\alpha, \beta\}}^{\ell} \leq M^{\ell}$. Since $T_{\{\alpha, \beta\}}$ is maximal in $L$, it follows that $T_{\{\alpha, \beta\}}$ is the only possibility for $M$, since otherwise $M^{\ell}$ would equal $L^{\ell}$, giving a contradiction by way of Lemma 3.3.

CASE $T=\operatorname{PSU}(3, q), m=q^{3}+1$ WITH $q \geq 3$ : Here $L$ corresponds to a transitive decomposition occurring in one of Cases 2,8 or 10 of Table 3 . Again, the transitive decomposition refines a decomposition corresponding to a 2 -transitive linear space $\mathcal{D}$, and we have

$$
T_{(\alpha, \beta)}^{\ell} \leq M^{\ell} \unlhd L^{\ell} \leq T_{\ell}^{\ell}
$$


where $\ell$ is the line of $\mathcal{D}$ containing $\alpha$ and $\beta$. In Case 2 we can apply Lemma 2.5 and argue as we did for $T=\operatorname{PSL}(a, q)$ to find that $M^{\ell}=L^{\ell}$, which contradicts Lemma 3.3. Suppose we are now in Case 8 . When $T=\operatorname{PSU}(3,3)$ we have $T_{\ell}^{\ell}=$ $P G U(2,3)=\operatorname{PGL}(2,3)$, and $L^{\ell}$ is as in Case 6 . Hence, by our treatment of Case 6 we obtain Line (v) of Table 1 . When $T=\operatorname{PSU}(3,5)$ we have $T_{\ell}^{\ell}=$ $P G U(2,5)=\operatorname{PGL}(2,5)$, and $L^{\ell}$ is as in Case 7 ; again giving a contradiction with Lemma 3.3. Now assume we are in Case 10. Then $L$ is a maximal subgroup of $T$ of order 96 . A consequence of [15, Theorem 6] is that $T_{\{\alpha, \beta\}}$ is maximal in $L$, and so by Lemma 3.2, a proper normal subgroup of $L$ containing $T_{(\alpha, \beta)}$ is either $T_{(\alpha, \beta)}$ or an index 2 subgroup of $L$. We checked using Magma that neither of these possibilites can occur. Hence $M=L$, which is a contradiction.

CASE $T={ }^{2} G_{2}(q), m=q^{3}+1$ WITH $q=3^{2 c+1}>3$ : Here we are in Case 2 of Table 3. Once again, applying Lemma 2.5 and arguing as we did for $T=\operatorname{PSL}(a, q)$ we obtain a contradiction with Lemma 3.3. (We examine $T={ }^{2} G_{2}(3) \cong \operatorname{P\Gamma L}(2,8)$ separately at the end of the proof.)

CASE $T=\operatorname{Sp}(2 l, 2)=S p(2 l, 2), m=2^{2 l-1} \pm 2^{l-1}$ : $\quad$ Recall from Section 2.3 the description of the $T$-transitive decomposition $\left(K_{m}, \mathcal{Q}\right)$ from Example 9 of [15]. Assume that $L=T_{Q_{v}}$ for some $v=a+b \in V \backslash\{0\}$, and recall that by Lemma 2.10, $T_{Q_{v}} \leq T_{v}$. Suppose that $M$ is a normal subgroup of $T_{v}$, and assume that $2 l \geq 8$. By Lemma 2.11, $T_{v}$ contains normal subgroups $Z$ and $N$ where $|Z|=2$ and $N / Z$ is the unique minimal normal subgroup of $T_{v} / Z$. Thus $M Z / Z$ either is trivial or contains $N / Z$. Since $T_{\left(\theta_{a}, \theta_{b}\right)} \leq M$ we must have $N / Z \leq M Z / Z$. Now $(M Z / Z) /(N / Z)$ is normal in $\left(T_{v} / Z\right) /(N / Z)$ which, by Lemma 2.11, is isomorphic to $S p(2 l-2,2)$ and is therefore simple since $2 l \geq 8$. So $(M Z / Z) /(N / Z)$ is either $\left(T_{v} / Z\right) /(N / Z)$ or trivial. In the former case $M Z / Z=T_{v} / Z$ and so either $M=T_{v}=L$ (which is a contradiction), or $\left|T_{v}: M\right|=2$ (and $Z \not \leq M$ ). But then $|N: M \cap N|=2$, with $(M \cap N) \triangleleft T_{v}$ and $Z \not \leq(M \cap N)$, contradicting Lemma 2.12. Hence $(M Z / Z) /(N / Z)$ must be trivial, meaning that $M \leq N$.

The size of $T_{\left(\theta_{a}, \theta_{b}\right)}$ is

$$
\frac{|T|}{\left(2^{2 l-1} \pm 2^{l-1}\right)\left(2^{2 l-1} \pm 2^{l-1}-1\right)}=\frac{\prod_{i=1}^{l}\left(2^{2 i}-1\right) 2^{2 i-1}}{\left(2^{2 l-1} \pm 2^{l-1}\right)\left(2^{2 l-1} \pm 2^{l-1}-1\right)}
$$

and it can be shown that this value is larger than $|N|=2^{2 l-1}$. Hence $M$ is not large enough to contain an arc stabiliser. So $M$ must equal $L$, which is a contradiction. We used MaGma to check that the result also holds for $2 l=6$.

CASE $T=A_{7}, m=15$ : Here $L$ is the stabiliser of a line $\ell$ of $\operatorname{PG}(3,2)$, and $L^{\ell}$ is permutationally isomorphic to $S_{3}$. Therefore $M^{\ell}$ must be either $A_{3}$ or $T_{(\alpha, \beta)}^{\ell}=1$, and hence we obtain Lines (xvi) and (xvii) of Table 1.

CASE $T=\operatorname{PCL}(2,8), m=28$ : We go through each line in turn of Table 4 . When $L=\mathrm{P} \Gamma \mathrm{L}(2,8), \operatorname{A\Gamma L}(1,8)$, or $\operatorname{AGL}(1,8)$, the possibilities listed for $M$ in Lines (ii)-(iv) of Table 2 are well known to be the only non-trivial normal subgroups. That each possibility for $M$ contains $T_{(\alpha, \beta)}$ follows from the fact that it contains $T_{\{\alpha, \beta\}}$. The unique minimal normal subgroup $S \cong \mathbb{Z}_{2}^{3}$ of $\operatorname{AGL}(1,8)$ is abelian, and so taking $L=S$, the possibilites for the normal subgroup $M$ are $T_{(\alpha, \beta)} \times \mathbb{Z}_{2} \cong \mathbb{Z}_{2}^{2}, T_{\{\alpha, \beta\}} \cong \mathbb{Z}_{2}^{2}$, and $T_{(\alpha, \beta)}$, giving Line (v). Next we consider the three possibilities with $L \cong A_{4} \times \mathbb{Z}_{2}$, namely $T_{\ell}, C_{1}$ and $C_{2}$. Let $\tau$ be an involution in $A_{4}$ and $\sigma$ the generator of the direct factor $\mathbb{Z}_{2}$. When $L=T_{\ell}$, $T_{(\alpha, \beta)}$ corresponds to the subgroup $\langle\sigma\rangle$. Hence the only possibilites for $M$ are $S$ (which corresponds to $V_{4} \times \mathbb{Z}_{2}$ ) and $T_{(\alpha, \beta)}$, giving Line (vi). When $L=C_{1}, T_{(\alpha, \beta)}$ corresponds 
to $\langle\tau\rangle$ and $M$ can be $D\left(\cong A_{4}\right), S\left(\cong V_{4} \times \mathbb{Z}_{2}\right)$ or $T_{\{\alpha, \beta\}}$ (corresponding to $\left.V_{4}\right)$, giving Line (vii). When $L=C_{2}, T_{(\alpha, \beta)}$ corresponds to $\langle\tau \sigma\rangle$ and the only possibility for $M$ is $S$ $\left(\cong V_{4} \times \mathbb{Z}_{2}\right)$, giving Line (viii). Finally, when $L=D \cong A_{4}, T_{(\alpha, \beta)}$ corresponds to $\langle\tau\rangle$ and the only possibility for $M$ is $T_{\{\alpha, \beta\}}$ (corresponding to $V_{4}$ ), giving Line (ix).

This completes the proof of Theorem 1.2.

\section{Partial Linear spaces}

A partial linear space is a set $\mathcal{V}$ of points together with a set $\mathcal{L}$ of (at least two) lines. Each line is a subset of points, and every pair of points lies in at most one line. We denote the partial linear space by the pair $(\mathcal{V}, \mathcal{L})$. A partial linear space is line transitive if there is a group of permutations of the points which preserves and transitively permutes the lines.

Lemma 5.1 of [1] shows that line transitive partial linear spaces are in one-to-one correspondence with transitive decompositions in which the subgraphs are complete. Thus the following theorem (which constitutes part of a result from [1]) gives a characterisation of a particular class of line transitive partial linear spaces.

Theorem 4.1. Let $(\Gamma, \mathcal{P})$ be a $G$-transitive decomposition where $|\mathcal{P}| \geq 2, \Gamma=K_{m} \times K_{m}$ and $G$ is a primitive rank 3 group of product action type. Assume that the subgraphs $\Gamma_{P}$ are complete. Then for some 2-transitive normal subgroup $T$ of $H$ there exists a $T$ transitive decomposition $\mathcal{T}:=\left(K_{m}, \mathcal{Q}\right)$ corresponding to a 2-transitive linear space such that $\mathcal{P}=\mathcal{P}(\mathcal{T}, \mathcal{R}, \varphi)$ (as in [1, Construction 2.10]) for some $\varphi$, where $\mathcal{R}$ is the partition of $A K_{m}$ in which each part contains only one arc.

We can read off the possibilities for $T, \mathcal{Q}$ and $\mathcal{R}$ from Table 1, yielding the following Corollary to Theorem 1.2. This gives a more explicit classification of the class of partial linear spaces described in Theorem 4.1. An equivalent result in proved by Devillers in [6].

Corollary 4.2. Let $T$ be a 2-transitive group which is either non-abelian and simple or $\mathrm{P} \Gamma \mathrm{L}(2,8)$ of degree 28. Suppose that $\mathcal{T}:=\left(K_{m}, \mathcal{Q}\right)$ is a $T$-transitive decomposition corresponding to a linear space, and let $\mathcal{R}$ be the partition of $A K_{m}$ in which each part contains only one arc. Assume that $\mathcal{T}$ and $\mathcal{R}$ satisfy the conditions of $[1$, Construction 2.10]. Then one of the following holds.

(i) $T=\mathrm{P} \Gamma \mathrm{L}(2,8), m=28$ and each $Q \in \mathcal{Q}$ induces a copy of $K_{4}$, or

(ii) $T=A_{7}, m=15$ and each $Q \in \mathcal{Q}$ induces a copy of $K_{3}$, or

(iii) $T=\operatorname{PSL}(a, 2)$ with $a \geq 3, m=2^{a}-1$ and each $Q \in \mathcal{Q}$ induces a copy of $K_{3}$.

\section{REFERENCES}

[1] John Bamberg, Geoffrey Pearce, and Cheryl E. Praeger. Transitive decompositions of graph products: rank 3 grid type. Journal of Group Theory. To appear.

[2] Eiichi Bannai and Tatsuro Ito. Algebraic combinatorics. I. The Benjamin/Cummings Publishing Co. Inc., Menlo Park, CA, 1984. Association schemes.

[3] Peter J. Cameron. SGDs with doubly transitive automorphism group. J. Graph Theory, 32(3):229233, 1999.

[4] Peter J. Cameron and Gábor Korchmáros. One-factorizations of complete graphs with a doubly transitive automorphism group. Bull. London Math. Soc., 25(1):1-6, 1993.

[5] J. H. Conway, R. T. Curtis, S. P. Norton, R. A. Parker, and R. A. Wilson. Atlas of finite groups. Oxford University Press, Eynsham, 1985. Maximal subgroups and ordinary characters for simple groups, With computational assistance from J. G. Thackray.

[6] Alice Devillers. A classification of finite partial linear spaces with a rank 3 automorphism group of grid type. In preparation.

[7] Leonard Eugene Dickson. Linear groups: With an exposition of the Galois field theory. with an introduction by W. Magnus. Dover Publications Inc., New York, 1958. 
[8] John D. Dixon and Brian Mortimer. Permutation groups, volume 163 of Graduate Texts in Mathematics. Springer-Verlag, New York, 1996.

[9] William M. Kantor. Homogeneous designs and geometric lattices. J. Combin. Theory Ser. A, 38(1):66-74, 1985.

[10] Cai Heng Li and Cheryl E. Praeger. Constructing homogeneous factorisations of complete graphs and digraphs. Graphs Combin., 18(4):757-761, 2002. Graph theory and discrete geometry (Manila, 2001).

[11] Tian Khoon Lim. Edge-transitive homogeneous factorisations of complete graphs. 2004. Ph.D. Thesis. The University of Western Australia.

[12] Michael E. O’Nan. Automorphisms of unitary block designs. J. Algebra, 20:495-511, 1972.

[13] Geoffrey Pearce. On transitive decompositions of disconnected graphs. Submitted.

[14] Geoffrey Pearce. Transitive decompositions of graphs and their links with geometry and origami. Submitted.

[15] Thomas Q. Sibley. On classifying finite edge colored graphs with two transitive automorphism groups. J. Combin. Theory Ser. B, 90(1):121-138, 2004.

School of Mathematics and Statistics, University of Western Australia, 35 Stirling Highway, Crawley, W.A., Australia 6009

E-mail address: geoffrey.pearce@graduate.uwa.edu.au 